\title{
La Lecture interactive d'albums pour les élèves du préscolaire ayant des difficultés langagières : une intervention prometteuse pour améliorer le vocabulaire
}

\author{
MARIE-PIER GODIN \\ Université du Québec à Montréal \\ LUCIE GODARD \\ Université du Québec à Montréal \\ NATHALIE CHAPLEAU \\ Université du Québec à Montréal \\ ANDRÉANNE GAGNÉ \\ Université du Québec à Montréal
}

\section{Résumé}

Cette étude évalue l'impact d'une adaptation de la Lecture interactive d'albums (Wasik \& Bond, 2001) sur le vocabulaire et sur d'autres habiletés langagières d'élèves du préscolaire ayant des difficultés langagières $(n=7)$. L'intervention (cinq semaines) combinait des méthodes d'apprentissage implicite et explicite à travers la lecture d'histoires. Un test $t$ à échantillons appariés montre une amélioration significative du vocabulaire réceptif et des réseaux sémantiques. Ainsi, l'étude met en lumière une dichotomie entre le développement des versants réceptif et expressif, et démontre la possibilité d'implanter une intervention en vocabulaire en contexte de classe.

\section{Interactive Book Reading for Children with Language Impairment at Kindergarten: A Promising Intervention to Improve Vocabulary}

\section{Abstract}

This study aims to measure the impact of an adaptation of the Interactive Book Reading (Wasik \& Bond, 2001) on vocabulary and other related language abilities of kindergarteners with language impairment $(\mathrm{LI})(\mathrm{n}=7)$. The intervention (5 weeks) involved indirect and direct instruction through book reading. A t-test for paired sample shows a significant improvement of receptive vocabulary and semantic networks. These results indicate a dichotomy between the production and the comprehension of words. Further directions for vocabulary intervention through book reading with LI children will be discussed in light of the results. 


\section{Introduction}

Les habiletés langagières orales occupent une place centrale dans l'apprentissage de l'écrit. En effet, c'est vers l'âge de cinq ans, une fois que les habiletés orales sont suffisamment développées, que l'enfant est prêt à amorcer l'apprentissage formel de l'écrit (Kaiser, Roberts, \& McLeod, 2011; Kamhi \& Catts, 1999). Le fonctionnement adéquat des habiletés langagières orales permet de produire ou de comprendre plus aisément un message écrit. À l'inverse, des habiletés lacunaires risquent de se refléter lors de l'apprentissage de l'écrit (Dalpé, St-Pierre, \& Lefebvre, 2011). Les habiletés langagières lacunaires peuvent découler de perturbations biologiques (ex.: une dysphasie) ou environnementales (ex. : une sous-stimulation langagière de la part du milieu familial) (Kaiser et al., 2011; Leonard, 2014).

Comme l'origine des difficultés langagières peut être multiple, la population d'enfants ayant de telles difficultés est extrêmement hétérogène. C'est au fil du développement langagier et des interventions mises en place qu'un diagnostic peut être établi quant à la nature et à la persistance des difficultés langagières. Malgré l'importance que revêt le diagnostic pour le cheminement scolaire ultérieur de l'enfant, il peut être ardu d'émettre un diagnostic précis avant l'âge de six ans puisque l'enfant est encore au tout début de son développement langagier (de Weck \& Marro, 2010). De manière générale, les critères de persistance et de résistance aux interventions guident le choix d'une scolarisation en classe de langage - une classe spéciale qui vise prioritairement le développement du langage oral (Samson, 1993). Lorsque les difficultés langagières persistent et résistent aux interventions, la fréquentation d'une classe de langage est considérée. D'ailleurs, au Québec, 66,40 \% des élèves dysphasiques sévères ne sont pas scolarisés en classe normale (MELS, 2008). Au sein des classes de langage, les élèves ont généralement un diagnostic de retard sévère de langage ou de trouble langagier, tel une dysphasie. Le retard de langage est défini comme étant un délai dans l'acquisition du langage au regard des étapes normales d'acquisition (Piérart, 2004). Il s'agit généralement d'un déficit relatif à la phonologie et à la syntaxe dont la sévérité des manifestations peut être variable (Delahaie, 2009). En revanche, la dysphasie est un trouble langagier affectant les versants expressif et/ou réceptif du langage (De Weck et Marro, 2010) et les manifestations de ce trouble doivent affecter en premier lieu le langage oral (Leonard, 2014). Ainsi, les difficultés langagières de ces élèves sont importantes et persistantes, et nécessitent un suivi spécialisé en classe de langage.

Malgré leur hétérogénéité, les élèves du préscolaire ayant un retard sévère de langage (RSL) ou un trouble langagier présentent tous un vocabulaire limité (Catts, 1997; McGregor, Oleson, Bahnsen, \& Duff, 2013). Comme le vocabulaire représente le cœur de la compréhension langagière orale et écrite (Marulis \& Neuman, 2010), l'apprentissage de la lecture est parsemé d'embuches diverses pour ces élèves (Vadasy \& Nelson, 2012). D'ailleurs, pour ce qui est de la lecture, il a été démontré que ceux-ci ont des difficultés en identification des mots écrits (Snowling, Bishop, \& Stothard, 2000) et en compréhension en lecture (Catts \& Kamhi, 1999; Kelso, Fletcher, \& Lee, 2007).

Le vocabulaire : un élément essentiel pour l'apprentissage de la lecture

Au préscolaire, le vocabulaire représente l'une des principales variables associées à la réussite en lecture (National Early Literacy Panel, 2008). Selon le modèle de Gough 
et Tunmer (1986), le Simple View of Reading, la compréhension en lecture est le résultat d'une interaction efficiente entre les habiletés d'identification des mots écrits (le décodage et la reconnaissance visuelle et automatique des mots écrits) et celles reliées à la compréhension linguistique (le processus par lequel les informations lexicales, relatives aux phrases et aux discours sont interprétées (Gough \& Tunmer, 1986)). Ainsi, l'apprenti lecteur doit développer et mobiliser simultanément plusieurs habiletés lui permettant d'interpréter et de comprendre un texte dans son ensemble (Laplante, 2011). Parmi celles-ci, le vocabulaire joue un rôle de premier plan (Tunmer \& Chapman, 2012). En effet, il est à la fois un indice prédictif de la compréhension en lecture (Kaiser et al., 2011; Neuman, 2011) et des habiletés d'identification de mots écrits (Kendeou, van den Broek, White, \& Lynch, 2009; Ouellette, 2006). Plus précisément, lors de l'entrée à l'école, il prédit les habiletés à lire des mots à la fin de la première année du primaire (Vadasy \& Nelson, 2012). Par conséquent, selon le Simple View of Reading, le vocabulaire influence à la fois la compréhension linguistique et les habiletés d'identification des mots écrits (Tunmer \& Chapman, 2012).

Les élèves ayant un vocabulaire restreint au début de leur scolarisation sont hautement à risque de présenter des difficultés en lecture durant leur cheminement scolaire (Kaiser et al., 2011; Neuman, 2011). Or, pour les élèves ayant un RSL ou une dysphasie, le développement du vocabulaire représente un défi de taille. Les recherches montrent que leur vocabulaire se développe significativement plus lentement que celui d'enfants du même âge sans difficulté langagière (Bishop, 1997; Gray, 2004; Hick, Joseph, Conti-Ramsden, Serratrice, \& Faragher, 2002; Nash \& Donaldson, 2005; Parsons, Law, \& Gascoigne, 2005) et que l'apprentissage de nouveaux mots est considérablement plus ardu (Hick et al., 2002; Nash \& Donaldson, 2005).

\section{L'apprentissage du vocabulaire}

Étant donné que les élèves ayant un RSL ou une dysphasie sont hautement à risque d'avoir des difficultés en lecture (Bishop \& Adams, 1990) et qu'ils ont un vocabulaire limité (Catts, 1997; McGregor et al., 2013), quelques recherches se sont penchées sur l'apprentissage du vocabulaire et sur les effets des interventions en vocabulaire.

Dans un contexte naturel, le vocabulaire s'apprend en grande partie de façon implicite, grâce à une exposition aux mots, sans que ceux-ci ne soient nécessairement enseignés (Cunningham, 2005; Cunningham \& Stanovich, 1998; Vadasy \& Nelson, 2012). Dans un contexte préscolaire, l'un des meilleurs moyens d'améliorer le vocabulaire est la lecture d'histoires à voix haute (Cunningham, 2005; Cunningham \& Stanovich, 1998). Toutefois, bien que ces méthodes d'apprentissage implicite soient bénéfiques pour la population générale, les élèves ayant un vocabulaire restreint, comme ceux ayant un RSL ou une dysphasie, répondent moins à ce type d'intervention que leurs pairs (Nash \& Donaldson, 2005; Pui Fong \& Windsor, 2010; Rice, Buhr, \& Nemeth, 1990; Steele \& Mills, 2011). En fait, dans un tel contexte, les élèves ayant d'importantes difficultés langagières ont besoin d'une exposition plus fréquente aux nouveaux mots pour les apprendre, contrairement à leurs pairs sans difficulté (Rice, Oetting, Marquis, Bode, \& Pae, 1994). Tout comme les dysphasiques, les élèves ayant un RSL profitent davantage de situations favorisant un apprentissage explicite en raison, entre autres, de leurs difficultés à inférer le sens des mots inconnus (Harris, Golinkoff, \& Hirsh-Pasek, 
2011). Ainsi, le recours à une méthode d'apprentissage explicite, soit l'enseignement de mots ciblés (Harris et al., 2011; Vadasy \& Nelson, 2012), et à une exposition fréquente à ceux-ci (Nash \& Donaldson, 2005; Rice et al., 1990; Riches, Tomasello, \& ContiRamsden, 2005) est nécessaire pour ces élèves. Néanmoins, cette méthode ne peut se faire qu'avec une quantité plus restreinte de mots et ne permet pas de développer un vaste vocabulaire (Nagy, 2005). C'est pourquoi il est de plus en plus recommandé de combiner les méthodes explicites (l'enseignement de mots ciblés) aux méthodes implicites (ex. : la lecture d'histoires à voix haute) afin de profiter des avantages de chacune et d'offrir un soutien efficace dans l'apprentissage de nouveaux mots (Marulis \& Neuman, 2010; Nash \& Donaldson, 2005; Neuman, 2011).

Parmi les interventions ciblant l'amélioration du vocabulaire au préscolaire, on retrouve l'Interactive Book Reading (Wasik \& Bond, 2001; Wasik, Bond, \& Hindman, 2006). Cette intervention s'appuie sur les principes d'une intervention efficace en vocabulaire (Graves, 2006; Stahl \& Fairbanks, 1986; Steele \& Mills, 2011), nommément, l'augmentation de la fréquence d'exposition aux mots ciblés, l'utilisation de contextes riches et variés et des discussions avec les élèves autour des caractéristiques sémantiques des mots. Cette intervention permet de joindre les méthodes d'apprentissage implicite et explicite dont la pertinence a été expliquée plus haut. La validation de cette approche a été faite auprès d'enfants âgés de trois à quatre ans vivant dans un quartier socioéconomique faible et non auprès d'élèves ayant d'importantes difficultés langagières (Wasik \& Bond, 2001; Wasik et al., 2006). À l'instar des élèves ayant un statut socioéconomique faible, les élèves ayant un RSL ou une dysphasie présentent un vocabulaire restreint. Ces élèves pourraient donc profiter des bienfaits de l'Interactive Book Reading (ci-après la Lecture interactive d'albums) tout comme les élèves provenant d'un milieu socioéconomique faible.

\section{Question de recherche}

À la lumière des informations précédentes, cette étude tente de répondre à la question suivante : Quel est l'impact de la Lecture interactive d'albums, une intervention favorisant l'apprentissage explicite et implicite de mots ciblés, sur le vocabulaire d'élèves du préscolaire ayant des difficultés langagières? Pour ce faire, la Lecture interactive d'albums (LIA) a été adaptée aux besoins de ces élèves et a été testée auprès d'une classe de langage du préscolaire au Québec.

\section{Cadre théorique}

Les élèves ayant d'importantes difficultés langagières et leur vocabulaire

En raison de l'hétérogénéité de la population ayant un RSL, il est difficile de déterminer la prévalence des retards langagiers (Kaiser et al., 2011). Selon des études américaines, le taux de prévalence se situe entre $7 \%$ et $13 \%$ (Tomblin et al., 1997; Zubrick, Taylor, Rice, \& Slegers, 2007). Au Québec, les élèves ayant une dysphasie représentent entre $1 \%$ et $7 \%$ de la population (Association québécoise pour les enfants atteints d'audimutité, 2006). L'une des principales difficultés des élèves ayant un RSL ou une dysphasie est l'apprentissage du vocabulaire (Bishop, 1997; Catts, 1997; McGregor et al., 2013). 
Le vocabulaire renvoie à l'ensemble de mots que connait une personne à l'oral ou à l'écrit. Il peut être examiné sous le versant réceptif du langage, soit les mots que l'on comprend, ou sous le versant expressif, soit les mots que l'on produit (Kamil \& Hiebert, 2005). Comme le soulève Leonard (2014), tous les élèves, y compris ceux ayant des difficultés langagières importantes, comprennent davantage de mots qu'ils peuvent en produire. Deux aspects du vocabulaire sont importants à considérer : la taille et la profondeur (Vadasy \& Nelson, 2012). D'une part, la taille (ou l'étendue) du vocabulaire renvoie au nombre de mots que connait une personne. En ce sens, les mots que la personne est en mesure de reconnaitre sont ajoutés au vocabulaire. Toutefois, la signification et l'utilisation de ces mots ne sont pas encore complètement maitrisées. D'autre part, la connaissance approfondie et l'utilisation appropriée des mots réfèrent plutôt à la profondeur du vocabulaire. La profondeur du vocabulaire se développe à la suite d'une exposition répétée aux mots et lorsque ceux-ci sont rencontrés dans différents contextes signifiants. Or, la profondeur fait davantage référence au versant expressif du langage, tandis que la taille du vocabulaire renvoie au versant réceptif (Vadasy \& Nelson, 2012). Les élèves ayant un RSL ou une dysphasie présentent des difficultés importantes en ce qui a trait au vocabulaire et ces dernières se manifestent autant au niveau de la profondeur que de la taille.

Sur le plan développemental, les enfants sans difficulté langagière connaissent une explosion lexicale vers l'âge de deux ans. Cette explosion ne se manifeste généralement pas chez les élèves ayant un RSL, particulièrement, chez ceux présentant une dysphasie (Leclercq \& Leroy, 2012). Ceux-ci auraient plutôt tendance à produire leurs premiers mots vers l'âge d'un an et onze mois comparativement aux enfants sans difficulté langagière qui les produisent vers l'âge de 11 mois (Leonard \& Deevy, 2004). Même à l'âge scolaire, le développement du vocabulaire chez les élèves ayant un RSL ou une dysphasie demeure significativement plus lent que celui d'enfants du même âge sans difficulté (Hick et al., 2002; Nash \& Donaldson, 2005).

Sur le plan quantitatif, la taille du vocabulaire est plus restreinte pour les élèves ayant un RSL ou une dysphasie (de Weck \& Marro, 2010; Leonard, Miller, \& Gerber, 1999). En effet, ils ont une diversité lexicale significativement inférieure à celles d'enfants du même âge chronologique sans difficulté langagière et à ceux plus jeunes et ayant un niveau linguistique similaire (de Weck \& Marro, 2010). Même après l'acquisition des premiers mots, les élèves ayant un RSL ou une dysphasie comprennent et utilisent un nombre plus restreint de mots que les élèves de leur âge sans difficulté. Par exemple, Thal et ses collègues (1999) rapportent que les enfants ayant un RSL produisent environ 426 mots différents vers l'âge de trois ou quatre ans alors que les enfants présentant un développement typique en produisent un nombre similaire à l'âge de deux ans.

La taille restreinte du vocabulaire influence la qualité et la précision des productions orales. En effet, les élèves ayant un RSL ou une dysphasie utilisent les mêmes mots et ceux-ci ont, bien souvent, une valeur générique, ce qui signifie qu'ils peuvent être utilisés dans différents contextes (de Weck \& Marro, 2010; Kaiser et al., 2011; Leclercq \& Leroy, 2012). De plus, leurs difficultés d'accès lexical risquent de teinter les productions orales par des pauses, des hésitations, des circonlocutions et des erreurs lexicales (Leclercq \& Leroy, 2012; Leonard, 2014). De Weck et Marro (2010) relèvent principalement quatre types d'erreurs lexicales : des confusions entre des mots 
appartenant à la même catégorie sémantique (ex. : dire pomme plutôt que orange), des remplacements de mots par d'autres ayant une valeur générique (ex. : dire fleur plutôt que marguerite), des substitutions de mots pour d'autres dénommant le tout pour une partie (ex. : utiliser le mot ordinateur plutôt que écran) et l'utilisation de définitions par l'usage (ex. : utiliser couper le bois plutôt que hache). En somme, les élèves ayant d'importantes difficultés langagières ont un développement lexical qui diffère de celui d'élèves sans difficulté langagière sur les plans quantitatifs et qualitatifs.

\section{Influence de certaines habiletés langagières sur le vocabulaire}

En plus de leur vocabulaire restreint, les élèves ayant un RSL ou une dysphasie présentent d'autres habiletés langagières lacunaires qui peuvent influencer le développement du vocabulaire. Dans le cadre de cette étude, nous avons ciblé les réseaux sémantiques, l'accès lexical et le traitement phonologique, car ces habiletés langagières sont connues pour avoir de grandes répercussions sur le développement du vocabulaire (de Weck \& Marro, 2010; Leclercq \& Leroy, 2012; Leonard, 2014; Leonard \& Deevy, 2004).

Les réseaux sémantiques. En mémoire, les connaissances lexicales ne représentent pas une simple suite pêlemêle d'items : elles s'organisent en réseaux sémantiques. En effet, la récupération en mémoire d'un mot se fait plus efficacement lorsque celui-ci se retrouve associé à un réseau sémantique élaboré où les liens entre les mots sont précis (Leonard, 2014; Leonard \& Deevy, 2004). Les élèves ayant un RSL ou une dysphasie sont en mesure d'organiser de façon appropriée les mots dans un réseau sémantique. Par contre, cette organisation sémantique est bien souvent entravée en raison du nombre limité d'items lexicaux, du nombre restreint de liens entre ces items et de la faiblesse de ces liens (de Weck \& Marro, 2010; Leonard \& Deevy, 2004). Ainsi, ces élèves accèdent et récupèrent difficilement les mots de leur vocabulaire (Leonard, 2014). Aussi, comme le réseau sémantique s'enrichit et se complexifie par l'apprentissage de mots nouveaux, les élèves ayant un RSL ou une dysphasie apprennent moins de caractéristiques sémantiques reliées aux mots comparativement aux élèves de leur âge (Alt, Plante, \& Creusere, 2004) créant ainsi un réseau clairsemé.

L'accès lexical. Afin de comprendre ou de produire un mot à l'oral, il est nécessaire que l'apprenant accède efficacement à la forme sonore du mot (représentations phonologiques) et au sens qui lui est associé (représentations sémantiques) (Dalpé, StPierre, \& Lefebvre, 2010; Snowling \& Hulme, 2008). Ensemble, les représentations phonologiques et sémantiques facilitent l'évocation lexicale (Snowling \& Hulme, 2008). Or, pour les élèves ayant un RSL ou une dysphasie, l'accès lexical est bien souvent lacunaire (de Weck \& Marro, 2010; Snowling \& Hulme, 2008). Leonard (2014) rapporte que ces élèves nomment plus lentement des items que leurs pairs, tel que mesuré par une tâche de dénomination rapide. Ainsi, même si le mot est encodé en mémoire, l'accès et la récupération se font plus difficilement. Par ailleurs, un accès lexical déficitaire peut découler d'un réseau sémantique clairsemé où la faiblesse des liens entre les mots nuit à l'accès aux informations encodées (Leonard, 2014; Leonard \& Deevy, 2004). Par conséquent, l'accès lexical est facilité non seulement par des représentations phonologiques et sémantiques précises, mais aussi par une organisation efficiente des mots dans un réseau sémantique bien défini et élaboré. 
Le traitement phonologique. La capacité à élaborer un système de représentations phonologiques à partir de l'entrée auditive renvoie au traitement phonologique (Dalpé et al., 2010). Cette habileté est principalement reliée au vocabulaire, puisque le traitement phonologique est activé chaque fois qu'un mot est entendu. Or, des limites sur le plan phonologique sont fréquentes chez les élèves ayant un RSL ou une dysphasie (Leclercq \& Leroy, 2012). Plusieurs hypothèses sont soulevées quant à leurs difficultés phonologiques. Tallal (1990) propose que ces lacunes soient engendrées par des difficultés dans le traitement des informations séquentielles rapides. Sussman (1993), pour sa part, indique que les lacunes phonologiques pourraient découler de difficultés à faire correspondre le signal acoustique avec les représentations phonologiques encodées en mémoire à long terme. Plus récemment, une hypothèse de sous-spécification des représentations phonologiques, découlant de difficultés à former et à maintenir en mémoire à long terme les représentations phonologiques, a été émise (Edwards \& Lahey, 1998; Maillart, Schelstraete, \& Hupet, 2004). Même si la cause demeure inconnue, des difficultés fréquentes en traitement phonologique sont relevées chez les élèves ayant un RSL ou une dysphasie et celles-ci interfèrent avec l'apprentissage du vocabulaire.

Considérant les défis importants que rencontrent les élèves ayant d'importantes difficultés langagières dans l'apprentissage du vocabulaire, il est nécessaire de se pencher sur les moyens les plus efficaces pour améliorer le vocabulaire, mais aussi d'identifier les habiletés langagières qui répondent à l'intervention.

\section{L'apprentissage $d u$ vocabulaire chez les élèves ayant d'importantes difficultés langagières}

Dans la vie courante, une grande partie du vocabulaire s'apprend de manière implicite. Il importe de souligner que les élèves ayant un RSL ou une dysphasie sont en mesure d'apprendre de nouveaux mots de cette façon. Par contre, cette méthode est nettement moins efficace que pour leurs pairs sans difficulté langagière (Nash \& Donaldson, 2005; Pui Fong \& Windsor, 2010; Rice et al., 1990; Riches et al., 2005). Dans un contexte d'apprentissage implicite, après une courte exposition à de nouveaux mots, les élèves parviennent difficilement à dégager le sens de ces mots et à les utiliser (Rice et al., 1990). Différentes hypothèses explicatives ont été avancées.

D'abord, les élèves ayant un RSL ou une dysphasie peuvent présenter des lacunes dans l'encodage et la récupération des informations sémantiques et phonologiques. L'association entre la forme phonologique et le sens est difficilement établie et mémorisée (Alt et al., 2004; McGregor, Newman, Reilly, \& Capone, 2002; Nash \& Donaldson, 2005; Snowling \& Hulme, 2008). C'est pourquoi ces élèves ont besoin d'une exposition fréquente et répétée aux mots pour les encoder précisément et pour accéder efficacement à ceux-ci (Fukkink, Blok, \& de Glopper, 2001).

Une autre hypothèse explicative stipule que l'apprentissage implicite est assuré par des habiletés langagières orales efficientes (Cain, Oakhill, \& Elbro, 2003; Steele \& Watkins, 2010). Par conséquent, les méthodes d'apprentissage implicite ne seraient pas optimales pour améliorer le vocabulaire chez les élèves ayant un RSL ou une dysphasie, puisqu'ils ont précisément des habiletés langagières orales déficitaires. Même à l'âge scolaire (9-10 ans), ces élèves ne profiteraient pas autant d'un contexte d'apprentissage implicite, comparativement à leurs pairs, en raison, entre autres, de leurs difficultés à inférer le sens de nouveaux mots (Harris et al., 2011; Steele \& Watkins, 2010). 
Conséquemment, les méthodes d'apprentissage implicite ne sont pas suffisantes pour améliorer le vocabulaire d'élèves ayant d'importantes difficultés langagières. Les études sur les interventions en vocabulaire permettent de relever certains principes qui pourraient améliorer la réponse à l'intervention des élèves ayant un RSL ou une dysphasie.

Augmenter la fréquence d'exposition. Afin que les élèves ayant un RSL ou une dysphasie puissent bénéficier des situations d'apprentissage implicite, l'augmentation de la fréquence d'exposition aux mots est nécessaire pour les comprendre en profondeur (Nash \& Donaldson, 2005; Rice et al., 1994; Riches et al., 2005). Une exposition répétée aux mots permet d'affiner les représentations phonologiques et sémantiques en y ajoutant des informations appropriées et en y éliminant les détails incorrects (Fukkink et al., 2001). Par conséquent, cela enrichit les réseaux sémantiques et favorise l'encodage, le stockage, la consolidation et l'accès lexical. En augmentant la fréquence d'exposition aux mots, cela permet de diminuer le retard entre les performances aux tests de vocabulaire des élèves ayant d'importantes difficultés langagières et celles des élèves sans difficulté du même âge (Pui Fong \& Windsor, 2010).

Cibler des mots. Que ce soit pour les élèves sans difficulté ou ceux ayant un RSL ou une dysphasie, tous les élèves améliorent davantage leurs connaissances lexicales lorsque les mots sont enseignés de manière explicite à l'aide de définitions (Nash \& Donaldson, 2005). Il est donc nécessaire de cibler certains mots qui seront enseignés et définis. Des mots de haute fréquence, des concepts sous-jacents à ces mots et des mots rares peuvent être choisis au regard des objectifs d'intervention (Beck, McKeown, \& Kucan, 2002). En raison du vocabulaire restreint des élèves du préscolaire ayant d'importantes difficultés langagières, des mots utiles et fréquents sont à privilégier.

Utiliser des contextes riches de sens et variés. Une simple exposition répétée aux définitions des mots ciblés n'est pas suffisante pour améliorer le vocabulaire : ces mots doivent être rencontrés dans différents contextes riches de sens permettant de développer en profondeur le vocabulaire (Vadasy \& Nelson, 2012). Un contexte riche réfère à une source d'exposition et d'apprentissage du vocabulaire à travers, bien souvent, un contexte langagier écrit (ex. : la lecture d'histoire partagée ou la lecture indépendante) (Brett, Rothlein, \& Hurley, 1996; Steele \& Mills, 2011). La lecture d'histoire devient ainsi une occasion de rencontrer, de définir et d'apporter des explications quant aux mots ciblés, et ce, dans un contexte riche (Steele \& Mills, 2011). D'ailleurs, Kiernan et Gray (1998) ont montré que l'apprentissage des mots à partir d'un contexte langagier favorise le développement du vocabulaire des élèves du préscolaire ayant une dysphasie.

De plus, l'utilisation de contextes riches doit être une occasion de définir et d'offrir des précisions quant aux mots. En effet, les élèves qui obtiennent des explications sur les mots, pendant la lecture, améliorent davantage leur vocabulaire réceptif (Elley, 1989) et les apprentissages se maintiennent dans le temps (Brett et al., 1996). Aussi, en diversifiant les contextes d'utilisation des mots ciblés, cela augmente la fréquence d'exposition et favorise l'apprentissage des mots.

En somme, les élèves ayant un RSL ou une dysphasie au préscolaire améliorent davantage leur vocabulaire lorsque les mots sont ciblés et rencontrés fréquemment dans 
différents contextes signifiants. C'est dans ce sens que les études scientifiques (voir la métaanalyse de Marulis \& Neuman, 2010) recommandent de combiner les méthodes d'apprentissage explicite de mots ciblés au contexte riche et signifiant des méthodes d'apprentissage implicite.

Intervenir par la Lecture interactive d'albums (LIA)

Wasik et Bond (2001) ont développé une méthode permettant de créer des occasions d'apprendre et de rencontrer fréquemment des mots ciblés dans un contexte riche, soit à travers la lecture d'histoires à voix haute. Cette intervention est constituée de quatre étapes. La première vise la présentation de mots ciblés à l'aide d'accessoires et des questions sont posées concernant sa nature et son utilité. À la deuxième étape, l'enseignant lit un album jeunesse. Pendant la lecture, il pose des questions aux élèves sur l'histoire et sur les mots ciblés. À la troisième étape, il engage une discussion autour de l'histoire lue. Enfin, la dernière étape est une activité complémentaire (ex. : jardinage, cuisine, bricolage) créant une occasion de consolider les apprentissages et de rencontrer les mots dans d'autres contextes. Ces quatre étapes ont lieu pendant la même semaine. Chaque semaine permet de travailler un thème spécifique à l'aide de deux livres abordant ce thème.

La LIA a été appliquée auprès de 121 enfants âgés de quatre ans et provenant d'un milieu socioéconomique faible (Wasik \& Bond, 2001). À la suite des 15 semaines d'intervention, les enfants du groupe expérimental ont amélioré significativement leur vocabulaire réceptif et expressif comparativement aux enfants qui n'ont pas reçu cette intervention. Ultérieurement, Wasik et son équipe (Wasik et al., 2006) ont appliqué de nouveau cette intervention auprès d'enfants âgés de trois ans et provenant d'un milieu socioéconomique faible. Les mêmes résultats ont été observés après les neuf mois d'intervention; les enfants du groupe expérimental performaient significativement mieux en vocabulaire réceptif et expressif que les enfants du groupe contrôle. Cependant, les études de Wasik et de ses collègues (Wasik \& Bond, 2001; Wasik et al., 2006) ont examiné précisément l'amélioration du vocabulaire réceptif et expressif des élèves. Les habiletés langagières sous-jacentes au vocabulaire, telles que les réseaux sémantiques, l'accès lexical et le traitement phonologique, n'ont pas été considérées. Conséquemment, nous ne savons pas quelle habileté langagière a permis l'amélioration du vocabulaire.

Ces études montrent la possibilité d'améliorer le vocabulaire d'enfants de milieux défavorisés, ayant un vocabulaire restreint, à l'aide de la LIA. En fait, cette intervention est un excellent exemple qui illustre la combinaison de pratiques pédagogiques favorisant l'apprentissage implicite et explicite, comme le recommandent plusieurs auteurs (Marulis \& Neuman, 2010; Nash \& Donaldson, 2005; Neuman, 2011). Ainsi, la présente étude propose d'adapter cette intervention auprès d'élèves francophones du préscolaire ayant des difficultés langagières. Deux objectifs régissent cette étude :

1. Évaluer l'impact de la LIA sur les mesures du vocabulaire réceptif et expressif auprès d'élèves du préscolaire ayant des difficultés langagières.

2. Évaluer l'impact de la LIA sur les mesures d'habiletés langagières reliées au développement du vocabulaire, soit les réseaux sémantiques, l'accès lexical et le traitement phonologique, auprès d'élèves du préscolaire ayant des difficultés langagières. 


\section{Sujets}

\section{Méthodologie}

Cette étude est réalisée auprès de sept sujets $(n=7$; cinq garçons, deux filles) âgés de quatre à six ans $(\mathrm{M}=$ cinq ans et un mois; min. = quatre ans et deux mois; $\max .=$ six ans et deux mois) et fréquentant une classe de langage. En raison de leur bas âge, ce ne sont pas tous les élèves qui ont un diagnostic de trouble langagier (de Weck \& Marro, 2010). Cependant, la plupart d'entre eux (5/7) ont un retard sévère de langage (RSL) et les autres, une dysphasie (2/7). Leurs difficultés langagières importantes nuisent à leur intégration en classe normale Les sujets de cette étude présentent également des difficultés associées, telles que des difficultés motrices $(n=4)$, attentionnelles $(n=5)$ et des limites cognitives $(n=2)$. L'hétérogénéité des difficultés est une caractéristique commune des classes de langage (Vergara, 2009) et de cette population (Kaiser et al., 2011).

\section{Instrumentation}

Dans le but d'évaluer l'impact de la LIA sur le vocabulaire et sur certaines habiletés langagières, plusieurs instruments de mesure sont nécessaires. Des tests standardisés en vocabulaire réceptif et expressif, en réseaux sémantiques, en accès lexical et en traitement phonologique sont utilisés avant et après les interventions.

Évaluation du vocabulaire. Le vocabulaire réceptif est évalué par l'Échelle de vocabulaire en images Peabody (ÉVIP) (Dunn, Thériault-Whalen, \& Dunn, 1993), avant et après les interventions. Ce test standardisé permet d'évaluer la taille du vocabulaire oral acquis par le participant. Pour y parvenir, il lui est demandé de choisir, parmi quatre images, celle qui illustre le mieux la signification du mot cible qui est prononcé par l'évaluateur.

Le vocabulaire expressif est mesuré par le Test de dénomination (TDen) (Dudley \& Delage, 1980). Dans ce test normalisé, le participant doit nommer les vingt-quatre images qui lui sont présentées. S'il nomme le mot cible au premier essai, il obtient trois points, au deuxième, deux points et au troisième, un point. À la suite de l'obtention de quatre zéros consécutifs, le test se termine et le nombre de points est comptabilisé.

Évaluation des réseaux sémantiques. Les réseaux sémantiques sont mesurés par deux sous-tests de l'Évaluation clinique des notions langagières (CELF) (Wigg et al., 2009). Le premier sous-test, Famille de mots (F), permet d'évaluer les versants réceptif (R) et expressif (E) de cette habileté. Plusieurs fiches, comprenant trois ou quatre images, sont présentées au participant. Ce dernier doit nommer les images qui vont bien ensemble (réceptif) et en expliquer la raison (expressif). Le deuxième sous-test, Association de mots (AM), permet d'évaluer uniquement le versant expressif des réseaux sémantiques. Les trois tâches de ce test permettent d'évaluer les connaissances de trois catégories sémantiques. Le participant dispose de 60 secondes pour nommer, autant que possible, des mots appartenant à une catégorie sémantique.

Évaluation de l'accès lexical. Deux tests de la trousse Bilan de santé : évaluation du développement pour la scolarité 5 à 6 ans (BSEDS, version 4.0; 2011) de Azzano et ses collègues (2011) sont utilisés pour évaluer l'accès lexical. Les deux tests 
Dénomination rapide demandent au participant de nommer le plus rapidement possible, dans un premier temps, des couleurs et, dans un second temps, des images. Puisque le temps de réponse est calculé, une diminution du score entre le prétest et le posttest signifie une amélioration des performances. Ces deux tâches de dénomination permettent de « repérer les troubles d'évocation lexicale (manque du mot) et des difficultés dans la programmation phonologique des mots. » (p. I.12).

Évaluation $d u$ traitement phonologique. Pour l'évaluation du traitement phonologique, le test Répétition de logatomes (RL), issu du BSEDS (2011), est utilisé. Ce test demande aux participants de répéter dix pseudomots évaluant ainsi sa capacité à discriminer les sons de la langue, à segmenter les sons en réception et à programmer ces sons avant leur articulation (Azzano et al., 2011).

\section{Déroulement}

Intervention. L'intervention consiste à appliquer la LIA auprès d'une classe de langage, durant cinq semaines, à raison de trois séances (de 40 à 60 minutes) par semaine. Quinze mots, considérés comme étant fréquents pour des enfants de la maternelle, ont été ciblés. Ces mots sont tirés du Test de production de vocabulaire oral (TPVO) (Godard, 2012) et regroupés par thème (voir tableau A1).

Plutôt que de répartir les quatre étapes en une semaine d'intervention, comme le font Wasik et ses collègues (Wasik \& Bond, 2001; Wasik et al., 2006), nous avons intégré les quatre étapes en une séance d'intervention et répété cette dernière trois fois par semaine. Considérant les difficultés langagières importantes des élèves ayant un RSL ou une dysphasie, cela leur permet de rencontrer plus fréquemment les mots ciblés et de diversifier les contextes d'utilisation des mots.

L'adaptation de la LIA comprend quatre phases: la phase d'activation des connaissances, la phase de réalisation, la phase de réinvestissement et une phase complémentaire (activité complémentaire). D'abord, une phase d'activation des connaissances débute la séance et permet de présenter le thème et les trois mots ciblés, pour la semaine, à l'aide d'objets et d'images. Des questions sont posées aux élèves et des définitions sont créées avec eux (ex. : Connais-tu cet objet? À quoi sert-il?). Ainsi, le vocabulaire est présenté explicitement et de façon décontextualisée dans cette première étape.

Puis, la phase de réalisation est entamée. Afin de mettre en contexte les trois mots ciblés, selon le thème choisi, la lecture d'une histoire à voix haute est faite. Les élèves sont invités à participer et à répondre aux questions qui leur sont posées (ex. : Peux-tu me montrer une image en lien avec le mot « parapluie »?). Parce que les définitions des mots ciblés sont revues, et ce, dans un contexte riche de sens, cette deuxième étape sollicite, à la fois, les apprentissages explicites et implicites.

Ensuite, la séance se poursuit par une phase de réinvestissement autour des trois mots ciblés. Un bref retour sur l'histoire et sur les définitions est fait (ex. : Quels mots avons-nous travaillés aujourd'hui? Que peux-tu faire avec une "pelle»?). Cette troisième étape sollicite davantage les apprentissages explicites.

Enfin, tel que le suggèrent Wasik et Bond (2001), des activités complémentaires (bricolages, activités d'identification, coloriages) concluent la séance (voir tableau A). 
Elles sont bénéfiques et nécessaires afin de maximiser la fréquence d'exposition dans des contextes variés (Graves, 2006; Nash \& Donaldson, 2005; Vadasy \& Nelson, 2012).

Mesure. Tant au prétest qu'au posttest, les sujets ont été rencontrés à tour de rôle dans un local fermé et silencieux afin de procéder à la passation des tests standardisés. Préalablement à la séance expérimentale, une phase d'observation a eu lieu pour recueillir des informations concernant les sujets et pour leur permettre de se familiariser avec l'expérimentatrice. Les instruments de mesure ont été passés avant et après les cinq semaines d'intervention et répartis sur une période de deux semaines (deux semaines pour la passation du prétest et deux semaines pour la passation du posttest). Enfin, comme l'intervalle de temps est court entre le prétest et le posttest (sept semaines), une habituation aux tests pourrait avoir eu lieu. Par contre, Ghorbani et Rahmandoost (2012) ont montré que la passation d'un même test demeure fiable dans un tel intervalle de temps. Ainsi, considérant les conclusions de ces auteurs et les difficultés langagières importantes des jeunes sujets de cette étude, une habituation aux tests est peu probable.

\section{Méthode d'analyse des résultats}

Les performances des sujets sont, d'abord, analysées au regard des normes des instruments de mesure utilisés. Une fois les scores bruts obtenus pour chacun des sujets, un score moyen de l'échantillon est calculé pour chaque test, et ce, pour le prétest et le posttest. Une analyse descriptive ainsi qu'une comparaison des moyennes sont effectuées afin de vérifier si une différence apparait entre les résultats obtenus avant et après les interventions pour le vocabulaire, pour les réseaux sémantiques, pour l'accès lexical et pour le traitement phonologique. Un test $t$, à échantillons appariés, est privilégié. Il est important de souligner que cette étude est réalisée auprès d'un petit échantillon $(n=7)$. L'utilisation du Mann-Whitney $U$ Test aurait pu être envisagée en raison du petit échantillon. Ce test statistique est considéré comme efficace pour comparer les moyennes d'une distribution non normale (Larson-Hall, 2010). Un procédé statistique d'analyse paramétrique tel que le test $t$ ne peut être réalisé que si les résultats obtenus respectent une distribution normale (Larson-Hall, 2010). Un test de normalité a donc été effectué pour chacune des mesures (prétest/posttest), ainsi que pour chacune des différences. Puisque l'indice de symétrie divisé par l'erreur standard de la symétrie indique un ratio inférieur à deux, et ce, pour chacune des mesures, la normalité de la distribution est vérifiée (Weinberg \& Abramowitz, 2002). Comme le test de normalité a permis de relever une distribution normale, pour l'ensemble des tests utilisés et pour la différence entre les deux temps de mesure, le test $t$ est privilégié. Comme l'a démontré De Winter (2013), il est possible d'utiliser un test $t$ à échantillons appariés même lorsque l'échantillon est inférieur à cinq sujets $(\mathrm{n} \leq 5)$, tant que la taille de l'effet demeure grande. Enfin, la taille de l'effet est mesurée et interprétée au regard des critères mis en lumière par Cohen (1988) et repris par Volker (2006) : taille de l'effet faible $(d=0,2)$; moyenne $(d=0,5)$; grande $(d=0,8)$.

\section{Résultats}

En vocabulaire réceptif, une différence significative est notée entre les résultats du prétest et du posttest (voir tableau 1). Ces résultats montrent également une taille de l'effet $(d=0,75)$ plutôt grande. En vocabulaire expressif, la comparaison des scores 
moyens bruts indique une différence non significative entre les deux temps de passation ainsi qu'une taille de l'effet $(d=0,31)$ faible.

Puis, en ce qui a trait aux réseaux sémantiques sur les plans réceptifs et expressifs, les tests $t$ indiquent une différence significative entre les résultats obtenus au prétest et au posttest, et ce, pour tous les instruments de mesure utilisés (voir tableau 2). De plus, la taille de l'effet est considérée comme grande pour chacune des comparaisons des scores moyens bruts.

Tableau 1

Résultats obtenus au prétest et au posttest pour l'évaluation du vocabulaire

\begin{tabular}{|c|c|c|c|c|c|c|}
\hline $\begin{array}{c}\text { Mesures } \\
\text { vocabulaire }\end{array}$ & $\begin{array}{c}\text { Prétest } \\
\qquad \begin{array}{c}M \\
(\hat{E} T)\end{array}\end{array}$ & $\begin{array}{c}\text { Posttest } \\
M \\
(\dot{E} T)\end{array}$ & $t$ & $d d l$ & $p$ & $\begin{array}{c}d \text { de } \\
\text { Cohen }\end{array}$ \\
\hline $\begin{array}{l}\text { Vocabulaire réceptif } \\
\text { (ÉVIP) }\end{array}$ & $\begin{array}{c}\mathbf{n}=7 \\
19,57 \\
(9,676)\end{array}$ & $\begin{array}{c}\mathbf{n}=7 \\
26,29 \\
(8,301)\end{array}$ & 3,490 & 6 & $0,013^{*}$ & 0,75 \\
\hline $\begin{array}{l}\text { Vocabulaire } \\
\text { expressif (TDen) }\end{array}$ & $\begin{array}{c}20,14 \\
(8,611)\end{array}$ & $\begin{array}{c}23,343 \\
(11,631)\end{array}$ & 1,803 & 6 & 0,121 & 0,31 \\
\hline
\end{tabular}

Note. $\mathrm{M}=$ score moyen brut; ÉT = Écart-type; $\mathrm{n}=$ population; $\mathrm{ddl}=$ degré de liberté

$* p<.05 * * p<.01$

Tableau 2

Résultats obtenus au prétest et au posttest pour l'évaluation des réseaux sémantiques

\begin{tabular}{|c|c|c|c|c|c|c|}
\hline $\begin{array}{l}\text { Mesures } \\
\text { réseaux } \\
\text { sémantiques }\end{array}$ & $\begin{array}{c}\text { Prétest } \\
\qquad \begin{array}{c}M \\
(E ́ T) \\
n=6\end{array} \\
\end{array}$ & $\begin{array}{c}\text { Posttest } \\
\begin{array}{c}M \\
(\dot{E} T) \\
\mathrm{n}=6\end{array} \\
\end{array}$ & $t$ & $d d l$ & $p$ & $\begin{array}{c}d \text { de } \\
\text { Cohen }\end{array}$ \\
\hline $\begin{array}{l}\text { Réseaux sémantiques } \\
\text { réceptifs } \\
(\mathrm{CELF-FR})\end{array}$ & $\begin{array}{c}13,00 \\
(3,742)\end{array}$ & $\begin{array}{c}15,67 \\
(3,670)\end{array}$ & 2,794 & 5 & $0,038^{*}$ & 0,72 \\
\hline $\begin{array}{l}\text { Réseaux sémantiques } \\
\text { expressifs } \\
(\mathrm{CELF-FE})\end{array}$ & $\begin{array}{c}2,83 \\
(2,317)\end{array}$ & $\begin{array}{c}7,33 \\
(3,386)\end{array}$ & 5,316 & 5 & $0,003 * *$ & 1,55 \\
\hline $\begin{array}{l}\text { Réseaux sémantiques } \\
\text { total } \\
\text { (CELF-F-total) }\end{array}$ & $\begin{array}{c}15,83 \\
(5,707)\end{array}$ & $\begin{array}{c}23,33 \\
(5,888)\end{array}$ & 7,097 & 5 & $0,001 * *$ & 1,29 \\
\hline $\begin{array}{l}\text { Réseaux sémantiques } \\
\text { expressifs } \\
\text { (CELF-AM) }\end{array}$ & $\begin{array}{c}7,50 \\
(5,089)\end{array}$ & $\begin{array}{c}13,67 \\
(3,670)\end{array}$ & 3,757 & 5 & $0,013^{*}$ & 1,39 \\
\hline
\end{tabular}

Note. $\mathrm{M}=$ score moyen brut; ÉT = Écart-type; $\mathrm{n}$ = population; ddl = degré de liberté

$* p<.05 * * p<.01$ 
Ensuite, pour ce qui est de l'évaluation de l'accès lexical, les résultats obtenus aux deux tests ne montrent aucune différence significative entre le prétest et le posttest (voir tableau 3). De plus, la taille de l'effet pour le test de dénomination de couleurs est petite $(d=-0,23)$ et pour le test de dénomination d'images est plutôt moyenne $(d=-0,43)$. Enfin, pour ce qui est de la mesure du traitement phonologique, une différence non significative est relevée entre le prétest et le posttest (voir tableau 4). Toutefois, les résultats indiquent que la taille de l'effet est grande $(d=0,88)$.

Tableau 3

Résultats obtenus au prétest et au posttest pour l'évaluation de l'accès lexical

\begin{tabular}{|c|c|c|c|c|c|c|}
\hline $\begin{array}{c}\text { Mesures } \\
\text { accès lexical }\end{array}$ & $\begin{array}{c}\text { Prétest } \\
\qquad \begin{array}{c}M \\
(E ́ T)\end{array}\end{array}$ & $\begin{array}{c}\text { Posttest } \\
\qquad \begin{array}{c}M \\
(\hat{E} T)\end{array}\end{array}$ & $t$ & $d d l$ & $p$ & $\begin{array}{c}d \text { de } \\
\text { Cohen }\end{array}$ \\
\hline & $n=6$ & $n=6$ & & & & \\
\hline $\begin{array}{l}\text { Accès lexical } \\
\text { (BSEDS couleurs) }\end{array}$ & $\begin{array}{c}39,67 \\
(20,057)\end{array}$ & $\begin{array}{c}35,85 \\
(11,583)\end{array}$ & $-0,920$ & 5 & 0,400 & $-0,23$ \\
\hline $\begin{array}{l}\text { Accès lexical } \\
\text { (BSEDS images) }\end{array}$ & $\begin{array}{c}56,33 \\
(20,176)\end{array}$ & $\begin{array}{c}48,00 \\
(18,166)\end{array}$ & $-1,269$ & 5 & 0,260 & $-0,43$ \\
\hline
\end{tabular}

Note. $\mathrm{M}=$ score moyen brut; ÉT = Écart-type; $\mathrm{n}=$ population; $\mathrm{ddl}=$ degré de liberté

$* p<.05 * * p<.01$

Tableau 4

Résultats obtenus au prétest et au posttest pour l'évaluation du traitement phonologique

\begin{tabular}{lccccccc}
\hline \multicolumn{1}{c}{$\begin{array}{c}\text { Mesure } \\
\text { traitement } \\
\text { phonologique }\end{array}$} & $\begin{array}{c}\text { Prétest } \\
\boldsymbol{M}\end{array}$ & $\begin{array}{c}\text { Posttest } \\
(\dot{\boldsymbol{E}} \boldsymbol{T})\end{array}$ & $\begin{array}{c}\boldsymbol{M} \\
(\dot{\boldsymbol{E}} \boldsymbol{T})\end{array}$ & $\boldsymbol{n}=\mathbf{t}$ & $\boldsymbol{d d l}$ & $\boldsymbol{p}$ & $\begin{array}{c}\boldsymbol{d} \text { de } \\
\text { Cohen }\end{array}$ \\
\hline $\begin{array}{l}\text { Traitement } \\
\text { phonologique }\end{array}$ & 1,57 & 3,14 & 2,091 & 6 & 0,081 & 0,88 \\
(BSEDS RL) & $(0,976)$ & $(2,340)$ & & & & \\
\hline
\end{tabular}

Note. $\mathrm{M}=$ score moyen brut; ÉT = Écart-type; $\mathrm{n}$ = population; $\mathrm{ddl}=$ degré de liberté

$* p<.05 * * p<.01$

En somme, les résultats obtenus aux neuf mesures permettent de constater que les scores moyens bruts obtenus au posttest sont supérieurs à ceux du prétest. Par contre, l'amélioration est considérée comme significative uniquement pour les tests évaluant le vocabulaire réceptif et les réseaux sémantiques réceptifs et expressifs. En revanche, les élèves n'auraient pas amélioré significativement leur vocabulaire expressif, leur accès lexical et leur traitement phonologique au regard des résultats obtenus à ces tests standardisés. 


\section{Discussion}

Les objectifs de cette étude sont d'évaluer l'impact de la LIA sur les mesures du vocabulaire et les habiletés langagières sous-jacentes (les réseaux sémantiques, l'accès lexical et le traitement phonologique) auprès de sept élèves du préscolaire ayant d'importantes difficultés langagières, soit un RSL ou une dysphasie. La comparaison des résultats obtenus au prétest et au posttest a permis de relever une amélioration du vocabulaire et de certaines habiletés langagières à la suite des cinq semaines d'intervention. En effet, une différence significative est relevée uniquement pour les tests évaluant le vocabulaire réceptif et pour les réseaux sémantiques durant cette période.

\section{Discussion au regard des particularités propres aux élèves ayant des difficultés langagières}

Considérant les difficultés langagières importantes des élèves, certaines de leurs caractéristiques ont pu influencer leurs apprentissages et les résultats obtenus. D'abord, contrairement aux résultats des études de Wasik et de ses collègues (Wasik \& Bond, 2001; Wasik et al., 2006), notre étude montre uniquement une amélioration du vocabulaire réceptif et non pas du vocabulaire réceptif et expressif, ce qui semble témoigner d'une amorce du processus d'acquisition du sens du mot. Par ailleurs, le versant expressif du langage (vocabulaire expressif et accès lexical) ne s'est pas amélioré significativement pendant la durée de l'intervention. Les sujets des études précédentes sont composés d'élèves ayant un vocabulaire restreint et provenant d'un milieu socioéconomique faible. Les sujets de notre étude présentent plutôt un vocabulaire limité en raison de leur RSL ou de leur dysphasie. L'amélioration non significative du vocabulaire expressif et de l'accès lexical met en lumière la persistance et la résistance des difficultés langagières.

Puis, les élèves ayant un RSL ou une dysphasie présentent des lacunes importantes d'accès lexical. Ainsi, il est possible que les sujets aient appris et encodé en mémoire de nouveaux mots au cours de l'intervention, mais la production de ceux-ci a été restreinte par leurs difficultés sur le plan de l'accès lexical. On peut donc penser que des amorces pour récupérer les mots en mémoire pourraient s'ajouter aux étapes d'enseignement pour ces élèves.

Enfin, il importe de relever que les sujets de cette étude présentent d'autres difficultés associées pouvant influencer le développement du vocabulaire et leurs performances aux différents tests. D'abord, les résultats indiquent que le traitement phonologique ne s'est pas amélioré pendant la durée de l'intervention. Plusieurs sujets $(\mathrm{n}=4)$ présentent des difficultés motrices qui peuvent avoir un impact sur les résultats observés, précisément sur le plan expressif. En effet, ces sujets présentent des difficultés phonétiques en raison de leur articulation lacunaire. Il devient difficile de savoir si les sujets ont bel et bien amélioré leur capacité à apprendre une nouvelle séquence phonologique - un problème commun aux élèves ayant d'importantes difficultés langagières (Gathercole \& Baddeley, 1990; Maillart \& Parisse, 2006) - puisque les difficultés motrices peuvent camoufler une possible amélioration du traitement phonologique évalué par un test expressif. Considérant la taille de l'effet plutôt grande $(d$ $=0,88)$, il est possible que cette habileté se soit améliorée. En revanche, le petit échantillon $(\mathrm{n}=7)$ et la courte durée de l'intervention n'ont pas permis d'obtenir des résultats significatifs. Éventuellement, l'utilisation d'un test mesurant le versant réceptif 
(ex. : comparaison de paires minimales (bain/pain)) serait à privilégier chez les élèves présentant à la fois des difficultés motrices et des lacunes en ce qui concerne le traitement phonologique.

De plus, la plupart des sujets $(n=5)$ présentent des difficultés attentionnelles. Comme le soulève Leonard (2014), les difficultés attentionnelles influencent l'acquisition du langage, mais les difficultés langagières peuvent, à leur tour, influencer les capacités attentionnelles. D'une part, des lacunes sur le plan de l'attention peuvent influencer les habiletés à discerner les détails d'un message oral entendu et ralentir le rythme d'acquisition du langage; d'autre part, les difficultés en compréhension langagière peuvent engendrer une certaine frustration et amener l'élève à moins prêter attention au message oral (Leonard, 2014). Ainsi, dans le cadre de l'étude, les difficultés attentionnelles des sujets peuvent avoir teinté non seulement les apprentissages, mais également leurs performances aux tests évaluant diverses habiletés langagières.

Finalement, quelques sujets $(\mathrm{n}=2)$ présentent des limites cognitives soupçonnées d'être une déficience intellectuelle légère. Les enfants ayant une déficience intellectuelle présentent un retard dans le développement langagier aussi et parfois plus important que celui dans le développement cognitif (Kaiser et al., 2011). Leurs difficultés langagières apparaissent dans les versants expressif et réceptif. La présence de limites cognitives peut influencer le développement langagier et particulièrement celui du vocabulaire (van der Schuit, Segers, van Balkom, \& Verhoeven, 2011) ce qui peut expliquer l'amélioration non significative de certaines habiletés langagières, dont le vocabulaire expressif.

\section{Discussion au regard de la durée de l'intervention}

Vadasy et Nelson (2012) stipulent que la connaissance précise et l'utilisation appropriée de nouveaux mots - la profondeur du vocabulaire - demandent une exposition répétée, et ce, sur une longue période de temps. Les études de Wasik et de ses collègues (Wasik \& Bond, 2001; Wasik et al., 2006) ont montré une amélioration à la fois du versant réceptif et expressif au terme de l'intervention. Considérant que leurs interventions étaient d'une durée de 15 semaines et de neuf mois, l'application de la LIA (cinq semaines) au sein de notre étude n'a probablement pas été assez longue pour développer significativement le versant expressif. L'effet bénéfique de l'intervention observé sur le plan réceptif met en lumière la possibilité d'améliorer la taille du vocabulaire sur une courte période d'intervention, mais il illustre aussi la nécessité d'une intervention plus longue pour noter une amélioration du vocabulaire expressif. En ce sens, Brett et ses collègues (1996) suggèrent que si l'apprenant est exposé régulièrement aux nouveaux mots et que des informations lui sont fournies pour constamment enrichir sa compréhension, ces mots risquent d'être appris en profondeur et les apprentissages se maintiendront dans le temps.

En somme, notre étude met en lumière une certaine dichotomie entre la réponse à l'intervention des versants réceptif et expressif. En effet, une intervention de courte durée, mais intensive, permet de développer le plan réceptif, mais elle demeure insuffisante pour développer le plan expressif. Cela montre l'importance d'évaluer, à la fois, les versants expressif et réceptif pour obtenir un portrait juste du développement du vocabulaire. 


\section{Discussion au regard de la structure de l'intervention}

En plus des caractéristiques des élèves et de la durée de l'intervention, les résultats obtenus peuvent être expliqués par la structure même de l'intervention. Premièrement, lors de la phase d'activation des connaissances, les mots cibles sont présentés à l'aide d'objets et d'images. Certains auteurs (Bloom, 2000; Nelson \& Shaw, 2002) affirment que l'utilisation d'accessoires n'est pas corrélée significativement à l'apprentissage du vocabulaire. Dans la même veine, les chercheurs ayant appliqué cette intervention (Wasik et al., 2006) relèvent que les objets ne sont pas nécessaires pour apprendre de nouveaux mots. Par contre, aucune de ces études n'avait des sujets ayant des difficultés langagières. Comme la compréhension du langage est déficitaire chez ces élèves, une définition donnée à l'oral peut s'avérer difficile à traiter et demeurer ambigüe et complexe pour ces élèves. Au cours des interventions, l'utilisation d'accessoires a permis de soutenir le discours des élèves. Plus précisément, ils ont utilisé les objets pour communiquer leur compréhension des mots sans recourir au langage oral. Par exemple, lorsqu'il a été demandé aux élèves à quoi servait le mot cible arrosoir, les élèves ne parvenaient pas à verbaliser son utilisation. Toutefois, certains d'entre eux ont été en mesure de montrer qu'ils comprenaient le sens du mot en mimant qu'ils arrosaient les plantes. Par conséquent, dans le cadre de cette étude, le recours à un support visuel pour définir, montrer, illustrer ou mimer les mots ciblés semble particulièrement utile pour les sujets. Il est certain que l'utilisation d'accessoires ne peut se faire qu'avec des mots qui peuvent être représentés concrètement et, donc, avec un ensemble plus restreint de noms communs, de certains adjectifs ou de verbes. D'ailleurs, comme l'apprentissage des verbes représente plus particulièrement un défi pour les élèves ayant des difficultés langagières, notamment pour ceux dysphasiques (Leonard \& Deevy, 2004), la LIA permet difficilement d'enseigner certains verbes en raison de leur faible potentiel d'être représentés concrètement à l'aide d'images ou d'objets (ex. : les verbes attributifs, tels que paraitre, être, devenir, sembler, etc.). La contribution d'un support visuel pour l'apprentissage du vocabulaire auprès d'élèves ayant des difficultés langagières devra être validée dans des études ultérieures.

Deuxièmement, malgré la courte durée de l'intervention et l'organisation sémantique lacunaire, les élèves sont parvenus à affiner les liens entre les mots, au regard des tests évaluant les réseaux sémantiques. Il est possible que cette amélioration soit due à une exposition répétée aux mots ciblés puisque, en augmentant la fréquence d'exposition aux mots, cela permet à l'apprenant d'ajouter des informations appropriées et d'éliminer les détails incorrects aux réseaux sémantiques (Fukkink et al., 2001). De plus, une autre hypothèse pour laquelle les élèves ayant des difficultés langagières ont amélioré leurs réseaux sémantiques réside dans le regroupement de mots par thème. En effet, pour ces élèves, le regroupement des mots ciblés par thème facilite les apprentissages (Leonard \& Deevy, 2004). Non seulement la création de thèmes permet d'apprendre les mots dans un contexte riche, mais cela permet aussi d'être exposé à d'autres mots découlant de ce thème, de renforcer les liens entre les mots ciblés et d'enrichir le réseau sémantique.

Troisièmement, l'intervention permet de rencontrer des mots ciblés dans un contexte riche (lecture d'histoires à voix haute) et de fournir des explications concernant ces mots pendant la lecture. Ainsi, la lecture et les explications fournies amènent un développement accru du versant réceptif du langage (Elley, 1989). Des activités 
complémentaires visant spécifiquement le versant expressif auraient probablement permis de mieux d'améliorer l'accès lexical et le vocabulaire pour lesquels aucune amélioration significative n'a été notée après l'intervention.

\section{Les limites}

Bien qu'une amélioration significative du vocabulaire réceptif et des réseaux sémantiques ait été relevée pendant la période d'intervention, il importe d'interpréter ces résultats avec prudence. Considérant le petit échantillon de cette étude, les résultats ne peuvent pas être généralisables à l'ensemble de la population ayant des difficultés langagières. Cependant, puisque très peu d'études se sont penchées sur les interventions en vocabulaire chez cette population (Cirrin \& Gillam, 2008; Steele \& Mills, 2011) et, plus largement, sur la prévention des difficultés relatives à la lecture (Kaiser et al., 2011), ces données contribuent à un domaine de recherche encore peu exploré.

Par ailleurs, en n'ayant aucun groupe contrôle, il est difficile d'affirmer que les résultats sont bel et bien dus à la LIA et non, par exemple, à la maturation des sujets ou aux autres interventions qui ont été réalisées en classe. Le contexte écologique de l'étude permet donc difficilement de contrôler les multiples variables associées à la classe de langage (enseignement, suivi spécialisé en orthophonie ou en ergothérapie, etc.). Toutefois, il montre la possibilité d'implanter facilement une intervention dans un contexte réel de classe en respectant les principes établis (l'augmentation de la fréquence d'exposition à des mots ciblés, l'utilisation de contextes riches et variés et des discussions autour des caractéristiques sémantiques des mots).

De plus, il importe de relever que les écarts-types sont plutôt grands, et ce, pour la plupart des résultats obtenus. Cela met en évidence l'hétérogénéité des manifestations langagières et des difficultés associées (motrices, attentionnelles et cognitives) des sujets de l'étude, ce qui est, à la fois, une caractéristique commune aux classes de langage (Samson, 1993; Vergara, 2009) et à cette population (Kaiser et al., 2011). Un plus grand échantillon probabiliste, une intervention de plus longue durée et une méthodologie expérimentale intégrant un groupe contrôle permettraient de valider les résultats de cette étude.

Enfin, il importe de souligner que l'amélioration significative du vocabulaire réceptif et des réseaux sémantiques ne signifie pas que les sujets ne présentent plus de retard en ce qui concerne ces habiletés. Les sujets ont amélioré significativement leurs scores par rapport à leurs propres performances, mais, lorsque nous comparons la différence entre leurs scores et la norme, bien que l'écart diminue, le retard ne disparait pas complètement. Cela met donc en lumière la persistance et la résistance des difficultés langagières, malgré une intervention intensive et spécifique mise en place (de Weck \& Marro, 2010).

\section{Conclusion}

Cette étude avait pour objectif d'évaluer l'impact d'une intervention favorisant les apprentissages implicites et explicites sur les mesures en vocabulaire, en réseaux sémantiques, en accès lexical et en traitement phonologique, auprès d'élèves du préscolaire ayant des difficultés langagières, soit un RSL ou une dysphasie. Pour y parvenir, la LIA a été utilisée. La comparaison des scores moyens bruts avant et après les cinq semaines d'intervention a montré qu'il est possible d'améliorer le vocabulaire 
réceptif et les réseaux sémantiques pendant la durée de l'intervention. Malgré ses limites, l'étude met en lumière le potentiel de cette intervention pour développer les habiletés langagières orales d'élèves ayant d'importantes difficultés langagières, pour montrer la dynamique des habiletés langagières dans l'apprentissage du vocabulaire et pour relever les apports différents sur les versants réceptif et expressif du vocabulaire.

Cette étude permet d'alimenter un domaine encore peu exploré : les interventions ciblant le vocabulaire chez des élèves du préscolaire ayant des difficultés langagières. Elle permet de mettre en lumière la possibilité et la nécessité d'approfondir les connaissances dans ce domaine. C'est pourquoi de futures études sont essentielles pour vérifier les effets à long terme de la LIA, pour comparer les résultats à un groupe contrôle (échantillon probabiliste) et pour appliquer les interventions sur une plus longue période.

\section{Références}

Alt, M., Plante, E., \& Creusere, M. (2004). Semantic Features in Fast-Mapping : Performance of Preschoolers With Specific Language Impairment Versus Preschoolers With Normal Language. Journal of Speech, Language \& Hearing Research, 47(2), 407-420.

Association québécoise de la dysphasie. (2006). 2014: http://www.aqea.qc.ca/fr/index.php

Azzano, V., Jacquier-Rioux, M., Lepaul, D., Lequette, C., Pouget, G., \& Zorman, M. (2011). Bilan de Santé Évaluation du Développement pour la scolarité 5 à 6 ans (version 4.0) (BSEDS 5-6-V-4.0).

Beck, I. L., McKeown, M. G., \& Kucan, L. (2002). Bringing words to life : Robust vocabulary instruction. New York: The Guilford Press.

Bishop, D. V. M. (1997). Uncommon understanding. Hove : Psychology Press.

Bishop, D. V. M., \& Adams, C. (1990). A Prospective Study of the Relationship between Specific Language Impairment, Phonological Disorders and Reading Retardation. Journal of Child Psychology and Psychiatry, 31(7), 1027-1050.

Blake, S. (2011). Superlapin. Paris : L'École des loisirs.

Bloom, P. (2000). How children learn the meaning of words. Cambridge MA : MIT Press.

Brett, A., Rothlein, L., \& Hurley, M. (1996). Vocabulary Acquisition from Listening to Stories and Explanations of Target Words. The Elementary School Journal, 96(4), 415-422.

Cain, K., Oakhill, J. V., \& Elbro, C. (2003). The ability to learn new word meanings from context by school-age children with and without language comprehension difficulties. Journal of Child Language, 30(03), 681-694.

Catts, H. W. (1997). The early identification of language-based reading disabilities. Language, Speech \& Hearing Services in Schools, 28(1), 86.

Catts, H. W., \& Kamhi, A. G. (1999). Classification of Reading Disabilities. In H. W. Catts \& A. G. Kamhi (Eds.), Language and Reading Disabilities (pp. 73-94). United States of America : Ally \& Bacon.

Cirrin, F. M., \& Gillam, R. B. (2008). Language Intervention Practices for School-Age Children With Spoken Language Disorders: A Systematic Review. Language, Speech \& Hearing Services in Schools, 39(1), S110-S137. 
Cohen, J. (1988). Statistical power analysis for the behavioral sciences. New Jersey: Erlbaum.

Cunningham, A. E. (2005). Vocabulary Growth Through Independent Reading and Reading Aloud to Children. In E. H. Hiebert \& M. L. Kamil (Eds.), Teaching and Learning Vocabulary (pp. 45-68). New Jersey : Lawrence Erlbaum Associates.

Cunningham, A. E., \& Stanovich, K. E. (1998). What Reading Does for the Mind. American Educator, 8-15.

Dalpé, V., St-Pierre, M.-C., \& Lefebvre, P. (2010). Habiletés mises en jeu dans la lecture et l'écriture et facteurs d'influence. In M.-C. St-Pierre, V. Dalpé \& C. Giroux (dir.), Difficultés de lecture et d'écriture: prévention et évaluation orthophonique auprès des jeunes (pp. 47-94). Québec: Presses de l'Université du Québec.

Dalpé, V., St-Pierre, M.-C., \& Lefebvre, P. (2011). Habiletés mises en jeu dans la lecture et l'écritre et facteurs d'influence. In M.-C. St-Pierre, V. Dalpé \& P. Lefebvre (dir.), Difficultés de lecture et d'écriture: Prévention et évaluation orthophonique auprès des jeunes (pp. 47-94). Québec : Presses de l'Université du Québec.

de Lestrade, A., \& Perrin, M. (2007). Le parapluie de madame Hô. Toulouse : Éditions Milan jeunesse.

de Pressensé, D. (2011). La maison d'Émilie. Paris : Casterman

de Weck, G., \& Marro, P. (2010). Les troubles du langage chez l'enfant: Description et évaluation. Issy-les-Moulineaux : Masson.

de Winter, J. C. F. (2013). Using the Student's $t$-test with extremely small sample sizes. Practical Assessment, Research \& Evaluation, 18(10).

Delahaie, M. (2009). L'évolution du langage de l'enfant. De la difficulté au trouble. Guide ressources pour les profesionnels. France.

Dorémus, G. (2001). Chien-saucisse: Éditions du Rouergue.

Dudley, J., \& Delage, J. (1980). Tests de langage - Dudley/Delage.

Dunn, L. M., Thériault-Whalen, C. M., \& Dunn, L. M. (1993). Échelle de vocabulaire Peabody (ÉVIP).

Duquennoy, J. (2012). Le fantôme de neige. Paris : Albin Michel Jeunesse.

Edwards, J., \& Lahey, M. (1998). Nonwords repetitions of children with specific language impairment: Exploration of some explanations for their inaccuracies. Applied Psycholinguistics, 19(2), 279-309.

Elley, W. B. (1989). Vocabulary acquisition from listening to stories. Reading Research Quarterly 24, 174-187.

Enckels, L. (1999). L'arrosoir de Gaspard. Hasselt : Mango jeunesse.

Florian, M., VanDerPloeg, N., \& Couvé, J. (2011). Youpi ya! Bruxelles : Alice Éditions.

Foccroulle, L., \& Masson, A. (2009). Le secret du potager. Namur : Mijade.

Fukkink, R. G., Blok, H., \& de Glopper, K. (2001). Deriving Word Meaning from

Written Context: A Multicomponential Skill. Language Learning, 51(3), 477-496.

Gathercole, S. E., \& Baddeley, A. D. (1990). Phonological Memory Deficits in Language Disordered Children: Is There a Causal Connection? Journal of Memory and Language, 29(3), 336.

Ghorbani, M. R., \& Rahmandoost, M. (2012). Higher Task-induced Involvement Load Enhances Students' EFL Vocabulary Learning (Vol. 3).

Godard, L. (2012). Test de Production du Vocabulaire Oral. 
Gough, P. B., \& Tunmer, W. E. (1986). Decoding, reading, and reading disability. Remedial and Special Education, 7, 6-10.

Graves, M. F. (2006). The Vocabulary Book : Learning \& Instruction New York : Teachers College Press.

Gray, S. (2004). Word Learning by Preschoolers With Specific Language Impairment : Predictors and Poor Learners. Journal of Speech, Language, and Hearing Research, 47(5), 1117-1132.

Gutman, A., \& Hallensleben, G. (2011). Gaspard et Lisa et les tomates disparues. Paris : Hachette jeunesse.

Harris, J., Golinkoff, R. M., \& Hirsh-Pasek, K. (2011). Lessons from the Crib for the Classroom : How Children Really Learn Vocabulary. In S. B. Neuman \& D. K. Dickinson (Eds.), Handbook of Early Literacy Research (Vol. 3, pp. 49-65). New York : The Guilford Press.

Hick, R. F., Joseph, K. L., Conti-Ramsden, G., Serratrice, L., \& Faragher, B. (2002). Vocabulary profiles of children with specific language impairment. Child Language Teaching and Therapy, 18(2), 165-180.

Kaiser, A. P., Roberts, M. Y., \& McLeod, R. H. (2011). Young Children with Language Impairments: Challenges in Transition to Reading. In S. B. Neuman \& D. K. Dickinson (Eds.), Handbook of Early Literacy Research (Vol. 3, pp. 153-171). New York : Guilford.

Kamhi, A. G., \& Catts, H. W. (1999). Reading Development. In H. W. Catts \& A. G. Kamhi (Eds.), Language and Reading Disabilities (pp. 26-49). United States of America : Allyn and Bacon.

Kamil, M. L., \& Hiebert, E. H. (2005). Teaching and Learning Vocabulary : Perspectives and Persistent Issues. In E. H. Hiebert \& M. L. Kamil (Eds.), Teaching and Learning Vocabulary : Bringing Research to Practice (pp. 1-23). New Jersey : Lawrence Erlbaum Associates.

Kelso, K., Fletcher, J., \& Lee, P. (2007). Reading Comprehension in Children with Specific Language Impairment: An Examination of Two Subgroups. International Journal of Language \& Communication Disorders, 42(1), 39-57.

Kendeou, P., van den Broek, P., White, M. J., \& Lynch, J. S. (2009). Predicting reading comprehension in early elementary school: The independent contributions of oral language and decoding skills. Journal of Educational Psychology, 101(4), 765778.

Kiernan, B., \& Gray, S. (1998). Word learning in a supported-learning context by preschool children with specific language impairment. Journal of Speech, Language, and Hearing Research, 41(1), 161-171.

L., F. (2011). Émilie, une fille qui décoiffe. Talents Hauts.

Laplante, L. (2011). L'évaluation diagnostique des difficultés d'apprentissage de la lecture. In M.-J. Berger \& A. Desrochers (dir.), L'évaluation de la littératie (pp. 139-174). Ottawa : Les Presses de l'Univeristé d'Ottawa.

Larson-Hall, J. (2010). A Guide to Doing Statistics in Second Language Research Using SPSS. New York : Routledge.

Leclercq, A.-L., \& Leroy, S. (2012). Introduction générale à la dysphasie : caractéristiques linguistigues et approches théoriques. In C. Mailart \& M.-A. 
Schelstraete (Eds.), Les dysphasies: de l'évaluation à la rééducation (pp. 5-25). Issy-les-Moulineaux : Elsevier Masson.

Leonard, L. B. (2014). Children with Specific Language Impairment (2e édition). United States of America : The MIT Press.

Leonard, L. B., \& Deevy, P. (2004). Lexical Deficits in Specific Language Impairment. In L. Verhoeven \& H. v. Balkom (Eds.), Classification of Developmental Language Disorders: Theoretical Issues and Clinical Implications (pp. 209-234). New Jersey : Lawrence Erlbaum Associates.

Leonard, L. B., Miller, C., \& Gerber, E. (1999). Grammatical Morphology and the Lexicon in Children With Specific Language Impairment. Journal of Speech, Language \& Hearing Research, 42(3), 678-689.

Leroy, J., \& Delaporte, B. (2012). Supercoquet. Toulouse : Milan.

Maillart, C., \& Parisse, C. (2006). Phonological deficits in French speaking children with SLI. International Journal of Language \& Communication Disorders, 41(3), 253274.

Maillart, C., Schelstraete, M.-A., \& Hupet, M. (2004). Phonological Representations in Children With SLI: A Study of French. Journal of Speech, Language \& Hearing Research, 47(1), 187-198.

Marulis, L. M., \& Neuman, S. B. (2010). The Effects of Vocabulary Intervention on Young Children's Word Learning: A Meta-Analysis. Review of Educational Research, 80(3), 300-335.

McGregor, K. K., Newman, R. M., Reilly, R. M., \& Capone, N. C. (2002). Semantic Representation and Naming in Children With Specific Language Impairment. Journal of Speech, Language \& Hearing Research, 45(5), 998.

McGregor, K. K., Oleson, J., Bahnsen, A., \& Duff, D. (2013). Children with developmental language impairment have vocabulary deficits characterized by limited breadth and depth. International Journal of Language \& Communication Disorders, 48(3), 307-319.

McPhail, D. (2000). La musique de Maurice. Tours : L'école des loisirs.

Milbourne, A., \& Cleyet-Merle, L. (2005). Dans le nid. Londres : Éditions Usborne ministère de l'Éducation du Loisir et du Sport (MELS). (2008). Déclaration des effectifs scolaires, 2005-2006 et 2006-2007.

Nagy, W. (2005). Why Vocabulary Instruction Needs to Be Long-Term and Comprehensive. In E. H. Hiebert \& M. L. Kamil (Eds.), Teaching and Learning Vocabulary (pp. 27-44). New Jersey : Lawrence Erlbaum Associates.

Nash, M., \& Donaldson, M. L. (2005). Word Learning in Children With Vocabulary Deficits. Journal of Speech, Language, and Hearing Research, 48(2), 439-458.

National Early Literacy Panel. (2008). Developing Early Literacy. Washington DC : National Institute for Literacy.

Nelson, K., \& Shaw, L. K. (2002). Developing a socially shared symbolic system. In E. Amsel \& J. P. Byrnes (Eds.), Language, literacy, and cognitive development: The development of symbolic communication (pp. 27-57). Mahwah : Lawrence Erlbaum Associates.

Neuman, S. B. (2011). The Challenge of Teaching Vocabulary in Early Education. In S. B. Neuman \& D. K. Dickinson (Eds.), Handbook of Early Literacy Research (Vol. 3, pp. 358-372). New York: The Guilford Press. 
Ouellette, G. P. (2006). What's meaning got to do with it: The role of vocabulary in word reading and reading comprehension. Journal of Educational Psychology, 98(3), 554-566.

Parsons, S., Law, J., \& Gascoigne, M. (2005). Teaching receptive vocabulary to children with specific language impairment: a curriculum-based approach. Child Language Teaching and Therapy, 21(1), 39-59.

Piérart, B. (2004). Les dysphasies chez l'enfant : un développement en délai ou une construction langagière différente ? Enfance, 56, 5-19.

Pui Fong, K., \& Windsor, J. (2010). Word Learning in Children With Primary Language Impairment: A Meta-Analysis. Journal of Speech, Language \& Hearing Research, 53(3), 739-756.

Rice, M. L., Buhr, J. C., \& Nemeth, M. (1990). Fast mapping word-learning abilities of language-delayed preschoolers. Journal of Speech and Hearing Disorders, 55, 3342.

Rice, M. L., Oetting, J. B., Marquis, J., Bode, J., \& Pae, S. (1994). Frequency of input effects on word comprehension of children with specific language impairment Journal of Speech \& Hearing Research, 37(1), 106-111.

Riches, N. G., Tomasello, M., \& Conti-Ramsden, G. (2005). Verb Learning in Children with SLI: Frequency and Spacing Effects. Journal of Speech,Language \& Hearing Research, 48(6), 1397-1411.

Ross, T. (2007). Je veux de la lumière! Londres : Gallimard jeunesse.

Samson, M. (1993). Les classes de langage à la CECM. Montréal.

Snowling, M. J., Bishop, D. V. M., \& Stothard, S. E. (2000). Is Preschool Language Impairment a Risk Factor for Dyslexia in Adolescence? Journal of Child Psychology and Psychiatry, 41(5), 587-600.

Snowling, M. J., \& Hulme, C. (2008). Learning to Read with a Language Impairment The Science of Reading: A Handbook (pp. 397-412). Blackwell Publishing Ltd.

Stahl, S. A., \& Fairbanks, M. M. (1986). The Effects of Vocabulary Instruction: A Model-Based Meta-Analysis. Review of Educational Research, 56(1), 72-110.

Steele, S. C., \& Mills, M. T. (2011). Vocabulary intervention for school-age children with language impairment: A review of evidence and good practice. Child Language Teaching and Therapy, 27(3), 354-370.

Steele, S. C., \& Watkins, R. V. (2010). Learning word meanings during reading by children with language learning disability and typically-developing peers. Clinical Linguistics \& Phonetics, 24(7), 520-539.

Sussman, J. E. (1993). Perception of formant transition cues to place of articulation in children with language impairments. Journal of Speech, Language \& Hearing Research, 36(6), 1286-1299.

Tallal, P. (1990). Fine-grained discrimination deficits in language-learning impaired children are specific neither to the auditory modality nor to speech perception. Journal of Speech, Language \& Hearing Research, 33(3), 616-617.

Thal, D. J., O'Hanlon, L., Clemmons, M., \& Fralin, L. (1999). Validity of a Parent Report Measure of Vocabulary and Syntax for Preschool Children with Language Impairment. Journal of Speech, Language, and Hearing Research, 42(2), 482496. 
Tomblin, J. B., Records, N. L., Buckwalter, P., Zhang, X., Smith, E., \& O'Brien, M. (1997). Prevalence of Specific Language Impairment in Kindergarten Children. Journal of Speech, Language, and Hearing Research, 40(6), 1245-1260.

Tunmer, W. E., \& Chapman, J. W. (2012). The simple view of reading redux: Vocabulary knowledge and the independent components hypothesis. Journal of Learning Disabilities, 45(5), 453-466.

Vadasy, P. F., \& Nelson, J. R. (2012). Vocabulary Instruction for Struggling Students. New York : Guilford.

van der Schuit, M., Segers, E., van Balkom, H., \& Verhoeven, L. (2011). How cognitive factors affect language development in children with intellectual disabilities. Research in Developmental Disabilities, 32(5), 1884-1894.

van Genechten, G. (2012). Petit poisson blanc. Namur : Éditions Mijade.

Vergara, A. R. (2009). Étude de l'efficacité des classes de langage dans la région de Montréal sur la réussite éducative des élèves dysphasiques sévères. (Maitrise mémoire), Université de Montréal, Montréal.

Volker, M. A. (2006). Reporting effect size estimates in school psychology research. Psychology in the Schools, 43(6), 653-672.

Wasik, B. A., \& Bond, M. A. (2001). Beyond the pages of a book: Interactive book reading and language development in preschool classrooms. Journal of Educational Psychology, 93(2), 243-250.

Wasik, B. A., Bond, M. A., \& Hindman, A. (2006). The effects of a language and literacy intervention on Head Start children and teachers. Journal of Educational Psychology, 98(1), 63-74.

Weinberg, S. L., \& Abramowitz, S. K. (2002). Data analysis for the behavioral sciences using SPSS. New York : Cambridge University Press.

Wigg, E. H., Secord, W. A., Semel-Mintz, E., Boulianne, L., \& Labelle, M. (2009). Évaluation clinique des notions langagières fondamentales - version pour francophones du Canada.

Zubrick, S. R., Taylor, C. L., Rice, M. L., \& Slegers, D. W. (2007). Late Language Emergence at 24 Months: An Epidemiological Study of Prevalence, Predictors, and Covariates. Journal of Speech, Language, and Hearing Research, 50(6), 1562-1592.

\section{Les biographies des auteures}

Marie-Pier Godin est étudiante au doctorat en éducation à l'Université du Québec à Montréal. Elle est titulaire d'une maitrise en didactique des langues et d'un baccalauréat en enseignement en adaptation scolaire et sociale. Ses travaux de recherche portent principalement sur l'enseignement et l'apprentissage de l'écrit chez les élèves en difficulté, particulièrement, chez ceux présentant des troubles langagiers.

Lucie Godard est professeure au Département de didactique des langues de l'Université du Québec à Montréal. Auparavant, elle a exercé l'orthophonie en milieu scolaire de nombreuses années. Ses recherches portent sur les liens entre le langage oral et le langage écrit principalement pour l'enseignement/apprentissage auprès des élèves à risque et en difficulté. Elle s'intéresse présentement à l'implantation du modèle à trois niveaux de réponse à l'intervention dans des milieux où les élèves sont plus vulnérables. 
Nathalie Chapleau est professeure au Département d'éducation et de formation spécialisées de l'UQAM. Ses travaux de recherche portent sur l'intervention auprès de l'élève présentant une dyslexie-dysorthographie. Plus précisément, sa recherche doctorale a permis de valider un programme d'intervention orthopédagogique misant sur la morphologie pour compenser les difficultés alphabétiques et orthographiques de lecteurs-scripteurs en difficulté.

Andréanne Gagné est professeure au Département de didactique des langues de l'Université du Québec à Montréal. Formée en orthopédagogie, en apprentissage des langues secondes et en sciences des troubles de la communication humaine, elle s'intéresse à l'émergence de la littératie chez les enfants présentant un développement typique ou atypique et chez les enfants bilingues. 


\section{Appendice}

\section{Tableau A}

Les thèmes, les albums, les mots cibles et les activités complémentaires utilisés

\begin{tabular}{|c|c|c|c|}
\hline Thèmes & Mots cibles & Albums & $\begin{array}{c}\text { Activités } \\
\text { complémentaires }\end{array}$ \\
\hline \multirow{3}{*}{ Les animaux } & \multirow{3}{*}{ lapin, poisson, nid } & Superlapin (Blake, 2011) & Découpage et coloriage \\
\hline & & $\begin{array}{l}\text { Petit poisson blanc } \\
\text { (van Genechten, 2012) }\end{array}$ & Relier les items semblables \\
\hline & & $\begin{array}{l}\text { Dans le nid } \\
\text { (Milbourne \& Cleyet-Merle, 2005) }\end{array}$ & $\begin{array}{l}\text { Suivre les consignes/ } \\
\text { identifier les mots cibles }\end{array}$ \\
\hline \multirow{3}{*}{$\begin{array}{l}\text { La maison } \\
\text { hantée }\end{array}$} & \multirow{3}{*}{$\begin{array}{l}\text { maison, fantôme, } \\
\text { lampe }\end{array}$} & $\begin{array}{l}\text { La maison d'Émilie } \\
\text { (de Pressensé, 2011) }\end{array}$ & Cherche et trouve \\
\hline & & $\begin{array}{l}\text { Le fantôme de neige } \\
\text { (Duquennoy, 2012) }\end{array}$ & Labyrinthe \\
\hline & & $\begin{array}{l}\text { Je veux de la lumière! } \\
\text { (Ross, 2007) }\end{array}$ & $\begin{array}{c}\text { Suivre les consignes/ } \\
\text { identifier les mots cibles }\end{array}$ \\
\hline \multirow{3}{*}{ La personne } & \multirow{3}{*}{$\begin{array}{l}\text { jupe, cheveux, } \\
\text { parapluie }\end{array}$} & $\begin{array}{l}\text { Supercoquet } \\
\text { (Leroy \& Delaporte, 2012) }\end{array}$ & Relier les items semblables \\
\hline & & $\begin{array}{l}\text { Émilie, une fille qui décoiffe } \\
\text { (L., 2011) }\end{array}$ & Découpage et coloriage \\
\hline & & $\begin{array}{l}\text { Le parapluie de madame Hô } \\
\text { (de Lestrade \& Perrin, 2007) }\end{array}$ & $\begin{array}{c}\text { Suivre les consignes/ } \\
\text { identifier les mots cibles }\end{array}$ \\
\hline \multirow{3}{*}{ Le jardin } & \multirow{3}{*}{$\begin{array}{l}\text { arrosoir, tomate, } \\
\text { pelle }\end{array}$} & $\begin{array}{l}\text { L'arrosoir de Gaspard } \\
\text { (Enckels, 1999) }\end{array}$ & Découpage et coloriage \\
\hline & & $\begin{array}{l}\text { Gaspard et Lisa et les tomates } \\
\text { disparues } \\
\text { (Gutman \& Hallensleben, 2011) }\end{array}$ & $\begin{array}{l}\text { Relier le mot cible à sa } \\
\text { fonction }\end{array}$ \\
\hline & & $\begin{array}{l}\text { Le secret du potager } \\
\text { (Foccroulle \& Masson, 2009) }\end{array}$ & $\begin{array}{c}\text { Suivre les consignes/ } \\
\text { identifier les mots cibles }\end{array}$ \\
\hline \multirow{3}{*}{ Les loisirs } & \multirow{3}{*}{$\begin{array}{l}\text { navire, violon, } \\
\text { saucisse }\end{array}$} & $\begin{array}{l}\text { Youpi ya! } \\
\text { (Florian, VanDerPloeg, \& Couvé, } \\
\text { 2011) }\end{array}$ & Découpage et coloriage \\
\hline & & $\begin{array}{l}\text { La musique de Maurice } \\
\text { (McPhail, 2000) }\end{array}$ & $\begin{array}{l}\text { Relier le mot cible à sa } \\
\text { fonction }\end{array}$ \\
\hline & & $\begin{array}{l}\text { Chien-saucisse } \\
\text { (Dorémus, 2001) }\end{array}$ & $\begin{array}{l}\text { Suivre les consignes/ } \\
\text { identifier les mots cibles }\end{array}$ \\
\hline
\end{tabular}

